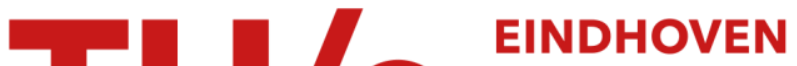 \\ UNIVERSITY OF \\ TECHNOLOGY
}

\section{Formation of fermionic molecules via interisotope Feshbach resonances}

\section{Citation for published version (APA):}

Kempen, van, E. G. M., Marcelis, B., \& Kokkelmans, S. J. J. M. F. (2004). Formation of fermionic molecules via interisotope Feshbach resonances. Physical Review A : Atomic, Molecular and Optical Physics, 70(5), 0507011/4. [050701]. https://doi.org/10.1103/PhysRevA.70.050701

DOI:

10.1103/PhysRevA.70.050701

Document status and date:

Published: 01/01/2004

\section{Document Version:}

Publisher's PDF, also known as Version of Record (includes final page, issue and volume numbers)

\section{Please check the document version of this publication:}

- A submitted manuscript is the version of the article upon submission and before peer-review. There can be important differences between the submitted version and the official published version of record. People interested in the research are advised to contact the author for the final version of the publication, or visit the $\mathrm{DOI}$ to the publisher's website.

- The final author version and the galley proof are versions of the publication after peer review.

- The final published version features the final layout of the paper including the volume, issue and page numbers.

Link to publication

\section{General rights}

Copyright and moral rights for the publications made accessible in the public portal are retained by the authors and/or other copyright owners and it is a condition of accessing publications that users recognise and abide by the legal requirements associated with these rights.

- Users may download and print one copy of any publication from the public portal for the purpose of private study or research.

- You may not further distribute the material or use it for any profit-making activity or commercial gain

- You may freely distribute the URL identifying the publication in the public portal.

If the publication is distributed under the terms of Article 25fa of the Dutch Copyright Act, indicated by the "Taverne" license above, please follow below link for the End User Agreement:

www.tue.nl/taverne

Take down policy

If you believe that this document breaches copyright please contact us at:

openaccess@tue.nl

providing details and we will investigate your claim. 


\title{
Formation of fermionic molecules via interisotope Feshbach resonances
}

\author{
E. G. M. v. Kempen, B. Marcelis, and S. J. J. M. F. Kokkelmans \\ Eindhoven University of Technology, P.O. Box 513, 5600 MB Eindhoven, The Netherlands
}

(Received 29 June 2004; published 19 November 2004)

\begin{abstract}
We perform an analysis of recent experimental measurements and improve the lithium interaction potentials. For ${ }^{6} \mathrm{Li}$ a consistent description can be given. We discuss theoretical uncertainties for the position of the wide ${ }^{6} \mathrm{Li}$ Feshbach resonance, and we present an analytic scattering model for this resonance, based on the inclusion of a field-dependent virtual open-channel state. We predict new Feshbach resonances for the ${ }^{6} \mathrm{Li}-{ }^{7} \mathrm{Li}$ system, and their importance for different types of crossover superfluidity models is discussed.
\end{abstract}

DOI: 10.1103/PhysRevA.70.050701 PACS number(s): 34.50.-s, 02.30.Mv, 03.75.Nt, 11.55.Bq

Resonances in cold atomic gases offer the key to connections with challenging condensed matter physics. In particular, resonances make lithium atomic systems very versatile. The first Bose-Einstein condensates (BEC) were quite small in number due to a negative scattering length [1]. Later Feshbach resonances [2] have been used to create condensates with positive scattering lengths and to generate bright solitons $[3,4]$ by changing the scattering length $a$ back to negative. Even more interesting is the usability to form molecules, since the Feshbach resonance results from bringing a molecular state on threshold. The connection between fermionic atoms and composite bosons (molecules) has great impact for the study of the well-known crossover problem between BEC and Bardeen-Cooper-Schrieffer (BCS)-type superfluidity [5-7].

In this Rapid Communication, we study several Feshbach resonances in the lithium system. We first review the knowledge of the interatomic interaction potentials, and use experimental data as input to improve these potentials. We discuss the special situation for the wide ${ }^{6} \mathrm{Li}$ resonance, where the background scattering length depends strongly on the magnetic field. This will be interpreted as a field-dependent virtual state (a second resonance), which is situated close to threshold. The full energy-dependent scattering process can be parametrized according to a simple analytical model that encapsulates both field-dependent resonances. Further, we apply our knowledge of the lithium interactions to a system of ${ }^{6} \mathrm{Li}^{7} \mathrm{Li}$, and find several Feshbach resonances that are accessible in current experimental setups. The underlying molecular state is of a composite fermionic nature, which allows for a new type of crossover physics - the transition of an atomic BEC to a molecular Fermi-type of superfluidity. Recently, Feshbach resonances in a heteronuclear BoseFermi mixture have been observed [8,9], where polar fermionic molecules underly the resonance state.

For an accurate prediction of resonance properties, we need a detailed understanding of the actual interatomic potentials. Here we describe how we improved the precision of existing potentials by using recent experimental measurements as input. The potentials can be divided in two radial intervals. For large interatomic separations $r$ the potential is given by the sum of the dispersive van der Waals tail, $V^{\mathrm{vdW}}(r)=-C_{6} / r^{6}-C_{8} / r^{8}-C_{10} / r^{10}$, and the exchange contribution, $V_{S}^{\mathrm{ex}}(r)=(-1)^{S+1} C^{e x} r^{7 / 2 \alpha-1} e^{-2 \alpha r}[10]$, resulting in two potentials: a singlet $(S=0)$ and a triplet $(S=1)$ potential. The coefficient $C^{e x}$ is taken from Refs. [11,12], $\alpha$ is directly related to the ionization energy $\alpha^{2} / 2$ [13], and $C_{8}$ and $C_{10}$ are taken from Ref. [14]. For smaller $r$ we use the model singlet and triplet potentials, which have also been used in Refs. $[15,16]$.

These short- and long-range potentials are smoothly connected at $r=18 a_{0}$, with $a_{0}$ the Bohr radius. To overcome the inaccuracies of the short-range potentials, we make use of the accumulated phase method [15]. A boundary condition is applied on the partial-wave radial wave functions at $r$ $=17.5 a_{0}$ in the form of a WKB phase $\phi_{S, T}(E, \ell)=\phi_{S, T}^{0}(E, \ell)$ $+\Delta \phi_{S, T}$. The first term on the right-hand side is calculated by radial integration of the model potential up to $17.5 a_{0}$ and is expected to account for the energy and angular momentum dependence of the accumulated phase to a sufficient degree of accuracy. The second term is an energy and angular momentum independent shift of the phase, determined from experimental data. These corrections $\Delta \phi_{S, T}$ to the accumulated singlet and triplet phases can be converted to the more physical quantities $\nu_{D S, D T}$, which are the fractional vibrational quantum numbers at dissociation.

We determine the free parameters of our interaction potentials $\nu_{D S}, \nu_{D T}$, and $C_{6}$ from experimental input by means of a $\chi^{2}$ minimization. An interisotope analysis, in which ${ }^{7} \mathrm{Li}$ is related to ${ }^{6} \mathrm{Li}$ by means of a simple mass-scaling relation, failed, yielding inconsistent results for $\nu_{D S}$. This is a strong indication of a breakdown of the Born-Oppenheimer approximation for the singlet potential. Such a breakdown was demonstrated in detailed spectroscopy [17]. We therefore avoid mass scaling of the singlet potential, and we perform two different analyses. In the first analysis, we only take ${ }^{6} \mathrm{Li}$ data into account. In the second analysis we investigate ${ }^{7} \mathrm{Li}$ as well; however, we only do a mass scaling for the triplet potential. Our total set of ${ }^{6} \mathrm{Li}$ experiments comprises six data points: the zero crossing of the scattering length of a system in the two lowest hyperfine states $[18,19]$; in the same spin state configuration, the positions of the narrow [20] and wide [7] Feshbach resonances; and the measurement of the scattering length in the lowest and third to lowest hyperfine state [21] and the binding energy of the most weakly bound triplet state [22].

In our first analysis we obtain a minimum in the reduced $\chi^{2}$ distribution of $\chi^{2}=0.5$. The corresponding parameter val- 


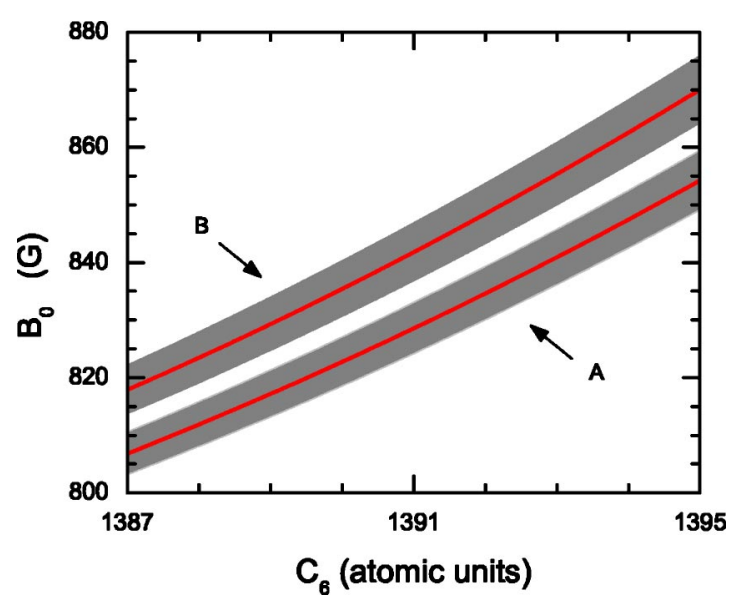

FIG. 1. (Color online) Dependency of the wide-resonance position $B_{0}$ on $C_{6}$, according to the second analysis (see text). The shaded area indicates the associated uncertainty. The curve indicated with A (B) is obtained making use of the exchange energy according to [11] ( [12] ).

ues are $\nu_{D S}=0.3496(5)$, equivalent with a singlet scattering length $a_{S}=45.3(1) a_{0}$ and $\nu_{D T}=0.9954(2)$, corresponding to $a_{T}=-2025(70) a_{0}$. For the leading dispersion coefficient we find $C_{6}=1388(6)$ a.u. This result has been obtained with a $C^{e x}$ of Ref. [12]. When we weaken $C^{e x}$ to the value of Ref. [11], we find that the optimal $C_{6}$ is shifted to $C_{6}=1390$ a.u. The scattering lengths found are consistent with previous determinations [16,22]. Our $C_{6}$ coefficient agrees with the values found in ab initio calculations [14,23].

The objective of a second analysis [24] is to reevaluate the position of the wide $s$-wave Feshbach resonance of ${ }^{6} \mathrm{Li}$ in the two lowest hyperfine states, without making use of the experimental result $B_{0}=822 \mathrm{G}$ [7]. Here we want to combine all available cold collision data on lithium, and we add the positions of three $p$-wave Feshbach resonances, which have been measured recently [25], and experimental data of ${ }^{7} \mathrm{Li}$ $[3,26]$, to the set of experiments. In this combined isotope analysis we perform a mass scaling procedure for the triplet boundary condition only. As explained above, we will not mass-scale the singlet potential but rather optimize the boundary conditions for the singlet potential independently for the two isotopes, by making $\nu_{D S, 7}$ a free parameter independent of $\nu_{D S, 6}$.

Since the $p$-wave resonances are measured with high accuracy, we also allow for small corrections of the angular momentum dependence of $\phi_{S}^{0}(E, \ell)$ via the parameter $\Delta \phi_{S}^{l}$ by means of an addition $\Delta \phi_{S}^{l} l(l+1)$, cf. [27]. By optimizing the interaction parameters $\left(\nu_{D S, 6}, \nu_{D S, 7}, \nu_{D T, 6}, \Delta \phi_{S}^{l}\right)$ for various fixed values of $C_{6}$, we are able to obtain a minimum reduced $\chi^{2}$ of 0.7 . The dependency of $\chi^{2}$ on $C_{6}$ is rather weak and therefore the set of experiments does not restrict the $C_{6}$ coefficient to an acceptable degree. However, the minimal $\chi^{2}$ occurs for $C_{6}=1390.6$ a.u. close to $a b$ initio values, when using $C^{e x}$ from Ref. [11], positioning the wide Feshbach resonance at $B_{0}=826 \mathrm{G}$. We estimate $808 \mathrm{G}$ $<B_{0}<846 \mathrm{G}$ for $1388<C_{6}<1393$ a.u. (see Fig. 1). For $C^{e x}$ from Ref. [12] we find a minimum $\chi^{2}=0.5$ for $C_{6}=1395.6$.

From now on, the properties of the Feshbach resonances will be derived from our first analysis. First, we study the

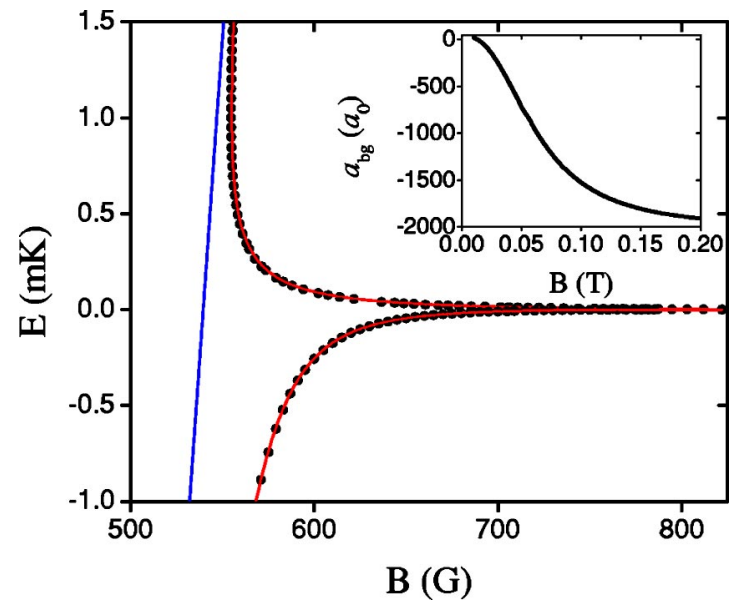

FIG. 2. (Color online) Energy of the dressed (quasi-)molecular state as obtained with a full coupled-channels calculation (dots), with respect to the $\left.\left.\left|f_{1}, m_{f_{1}}\right\rangle \otimes\left|f_{2}, m_{f_{2}}\right\rangle=1 / 2,1 / 2\right\rangle \otimes 1 / 2,-1 / 2\right\rangle$ threshold. The straight line is the bare closed-channel energy $\epsilon_{b}(B)$, and the energy of the dressed state according to our model is indicated by the curved line. Inset: corresponding background value of the scattering length as a function of the magnetic field $B$.

wide $B_{0}=822 \mathrm{G}$ Feshbach resonance in the two lowest hyperfine states of ${ }^{6} \mathrm{Li}$. This resonance is quite remarkable for two reasons: it has a large width of the order of $100 \mathrm{G}$, and its background scattering length $a_{\mathrm{bg}}$ is strongly dependent on the magnetic field, which can be seen from the inset of Fig. 2. At zero field, $a_{\mathrm{bg}}$ is 3 and positive, while for large field values $a_{\mathrm{bg}}$ is large and negative, indicating the presence of a nearby virtual state in the open-channel subspace $P$ [28]. Consequently several important quantities, such as the $S$ and $T$ matrices, which summarize the collision process, depend nontrivially on the collision energy $E$. Here we will apply the model discussed in Ref. [28] to this $s$-wave Feshbach resonance. This model takes the virtual state into account explicitly, and gives an analytical description of all the important two-body quantities near the Feshbach resonance.

In general, the relation between the background scattering length $a_{\mathrm{bg}}$, the range of the potential $a_{\mathrm{bg}}^{P}$, and the virtual state pole $\kappa_{\mathrm{vs}}$, is given by $a_{\mathrm{bg}}=a_{\mathrm{bg}}^{P}-1 / \kappa_{\mathrm{vs}}$. The range of the potential is related to the van der Waals coefficient $C_{6}$, and does not depend on the magnetic field. Therefore, we account for the field dependence of $a_{\mathrm{bg}}$ by generalizing the model of Ref. [28] to the case of a field-dependent virtual-state $\kappa_{\mathrm{vs}}(B)$. The complex energy shift is then given by

$$
A(E, B)=\Delta_{\mathrm{res}}(E, B)-\frac{i}{2} \Gamma(E, B)=\frac{-i A_{\mathrm{vs}}(B)}{2 \kappa_{\mathrm{vs}}(B)\left[k+i \kappa_{\mathrm{vs}}(B)\right]},
$$

where $A_{\mathrm{vs}}(B)$ is related to the coupling matrix element between the open-channel virtual state and the closed-channel bound state responsible for the Feshbach resonance. Our wave-number units are such that $E=k^{2}$.

The total scattering length is then given by 
TABLE I. Parameters of the virtual-state model. For the magnetic fields of interest $(530 \mathrm{G} \lesssim B \lesssim 830 \mathrm{G})$, the field-dependent parameters are given by a third-order polynomial fit, $c_{0}+c_{1} B+c_{2} B^{2}$ $+c_{3} B^{3}$. For $A_{\mathrm{vs}}(B)$ the units are given by $\left[c_{n}\right]=\mathrm{K}^{2} \mathrm{G}^{-n}$, and for $\kappa_{\mathrm{vs}}(B)$ the units are given by $\left[c_{n}\right]=\mathrm{K}^{1 / 2} \mathrm{G}^{-n}$. The range of the potential is given by $a_{\mathrm{bg}}^{P}=45 a_{0}$.

\begin{tabular}{ccccc}
\hline \hline & $c_{0}$ & $c_{1}$ & $c_{2}$ & $c_{3}$ \\
\hline$A_{\mathrm{vs}}(B)$ & $1.62 \times 10^{-5}$ & $-5.10 \times 10^{-8}$ & $6.07 \times 10^{-11}$ & $-2.56 \times 10^{-14}$ \\
$\kappa_{\mathrm{vs}}(B)$ & $4.65 \times 10^{-2}$ & $-1.54 \times 10^{-4}$ & $1.92 \times 10^{-7}$ & $-8.26 \times 10^{-11}$ \\
\hline \hline
\end{tabular}

$$
a(B)=a_{\mathrm{bg}}^{P}-\frac{1}{\kappa_{\mathrm{vs}}(B)}-\lim _{E \rightarrow 0} \frac{\Gamma(E, B) / 2}{k\left[\epsilon_{b}(B)+\Delta_{\mathrm{res}}(E, B)\right]},
$$

where $\epsilon_{b}(B)=\Delta \mu^{\mathrm{mag}}\left(B-\bar{B}_{0}\right)$ is the energy of the bare closedchannel bound state, $\Delta \mu^{\mathrm{mag}}=2.0 \mu_{B}$ the magnetic moment, $\mu_{B}$ the Bohr magneton, and $\bar{B}_{0}=539.5 \mathrm{G}$ the field where the bare closed-channel energy crosses threshold. For a fixed $B$ value, the parameters $A_{\mathrm{vs}}(B)$ and $\kappa_{\mathrm{vs}}(B)$ are obtained by fitting Eq. (2) to the coupled-channels result for $a(B)$, using two close-lying data points where we assume $A_{\mathrm{vs}}$ and $\kappa_{\mathrm{vs}}$ to be locally constant. Repeating this for every field value, we obtain explicit expressions for the nontrivial energy dependence of the complex energy shift, using only the zeroenergy information contained in $a(B)$. Note that there are only two free parameters, and $a_{\mathrm{bg}}(B)$ is fixed once $\kappa_{\mathrm{vs}}(B)$ is known.

The fit functions are summarized in Table I. In Fig. 2 we compare the dressed (quasi-)molecular state calculated by coupled-channels methods and by our analytical model, which agrees excellently. Therefore, this model can be used to analytically describe the $S$ and $T$ matrices [28], scattering phase shifts, etc., with similar precision as a full coupledchannels calculation, for a large range of energies and magnetic fields. We note also that the narrow $543 \mathrm{G}$ Feshbach resonance (width of order $0.1 \mathrm{G}$ ) can be described by Feshbach theory. Here, however, an easier description is possible based on only one single background part and a single resonance state, since the narrow resonance has a "local" background scattering length of the order of $a_{\mathrm{bg}}^{P}$. For narrow resonances [29,30], the typical resonance features in the continuum scattering, which depend on the details of the potential, are very important to the BEC-BCS crossover physics, since they are visible for energies less than the Fermi energy, resulting in a nonuniversal crossover picture.

Now we turn to the final topic of this paper, the study of Feshbach resonances in a ${ }^{6} \mathrm{Li}$-fermion- ${ }^{7} \mathrm{Li}$-boson mixture. We use again our first analysis of the interaction parameters for ${ }^{6} \mathrm{Li}$, and perform a simple mass scaling of the accumulated phases for the ${ }^{6} \mathrm{Li}^{-}{ }^{7} \mathrm{Li}$ system. As discussed before, this leads to less accurate predictions for the resonance positions, as suggested by the accuracies from the ${ }^{6} \mathrm{Li}$ interaction parameters. We estimate the inaccuracies in the mixed isotope resonance positions to be of order a few gauss, due to inaccuracies of the mass-scaling relations.

Feshbach resonances in the ${ }^{6} \mathrm{Li}^{-} \mathrm{Li}$ system have been studied before in Ref. [16], where only the case of magneti-

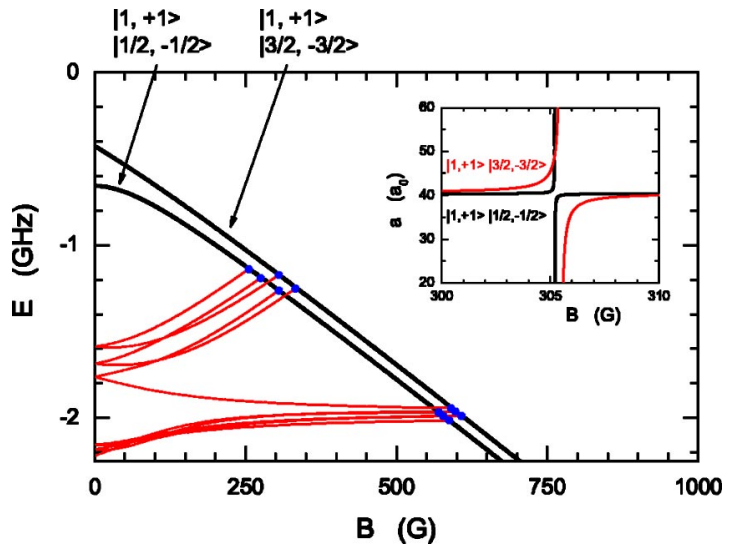

FIG. 3. (Color online) Two thresholds for the ${ }^{6} \mathrm{Li}-{ }^{7} \mathrm{Li}$ system, which show the coincidence of two Feshbach resonances. Inset: scattering length as a function of magnetic field, for the two coinciding Feshbach resonances.

cally trappable atoms was investigated. Moreover, those resonances are accompanied by large inelastic exchange losses. We investigate only ${ }^{6} \mathrm{Li}^{-}{ }^{7} \mathrm{Li}$ hyperfine state combinations where exchange losses are absent. Within these boundaries, there are still numerous resonances present, and we restrict ourselves to the most interesting results. The ${ }^{6} \mathrm{Li}-{ }^{7} \mathrm{Li}|1 / 2,1 / 2\rangle \otimes|1,1\rangle$ channel has the lowest energy in the two-body hyperfine diagram. Therefore, this channel will not suffer from magnetic dipolar relaxation. We find five Feshbach resonances at magnetic field values of 218, 230, 251, 551, and $559 \mathrm{G}$. Measurements of these resonances might provide the missing information to exactly locate the position of the wide ${ }^{6} \mathrm{Li}$ resonance, as all mixed resonances arise from the same underlying bound state in the triplet potential.

Feshbach resonances between bosons and fermions give rise to a fundamentally different type of crossover physics. Already some work on the interactions in Bose-Fermi mixtures can be found in the literature $[31,32]$. However, in order to describe correctly the many-body physics and interactions close to resonance, more research is needed. To make the system feasible, some requirements have to be fulfilled. For a stable BEC, a positive scattering length for the bosons is required. Approaching the resonance from the atomic side, the mixed boson-fermion scattering length will become negative, and stability of the system could become an issue. At the other side of the resonance, it is important that the effective interaction between the fermionic molecules is attractive. Pointlike composite fermions do not undergo $s$-wave collisions. However, close to resonance the molecules are long stretched, and an effective interaction mediated via the bosons could be possible. An approach similar to Ref. [33] could be conclusive on this and on the expected dependence of the inelastic rate coefficient on the scattering length. Also, the effect of Pauli blocking will not be as strong as in Ref. [33] since a three-body decay process with only one fermion and two bosons involved is possible. However, a reduction with respect to the pure bosonic case could still be expected.

Another interesting situation occurs when two Feshbach resonances coincide. Further away from resonance, where 
the size of the molecule is comparable to the size of the potential, $s$-wave collisions are not allowed for these composite fermions. Therefore, in order to preserve superfluid behavior in this region, two different molecular spin configurations are needed to allow for $s$-wave collisions between molecules. Moreover, it is desirable that the two resonances responsible for the molecule formation coincide. Such a coincidence can be found from Fig. 3, where the ${ }^{6} \mathrm{Li}-{ }^{7} \mathrm{Li} \mid 1 / 2$, $-1 / 2\rangle \otimes|1,1\rangle$ and $|3 / 2,-3 / 2\rangle \otimes|1,1\rangle$ bound states and thresholds are plotted as a function of magnetic field. Every crossing of a bound state with threshold indicates the position of a Feshbach resonance. It can be seen that at $B$ $=305 \mathrm{G}$ two Feshbach resonances coincide. These two threshold channels have the same bosonic ${ }^{7} \mathrm{Li}$ state, but a different fermionic ${ }^{6} \mathrm{Li}$ state. This coincidence is systematic, and will not depend on the interaction parameters. The scat- tering lengths as a function of the magnetic field can be seen in the inset of Fig. 3.

In conclusion, we analyzed recent experimental measurements for lithium, and showed that mass scaling between ${ }^{6} \mathrm{Li}$ and ${ }^{7} \mathrm{Li}$ fails for the singlet potential. We investigated uncertainties in the wide ${ }^{6} \mathrm{Li}$ resonance position, and demonstated an analytical model for this resonance that includes the nearby virtual state. Finally, we showed that ${ }^{6} \mathrm{Li}-{ }^{7} \mathrm{Li}$ mixtures feature accessible Feshbach resonances, giving rise to fermionic molecules, yielding new BCS-BEC crossover physics.

We acknowledge discussions with B. Verhaar, M. Holland, J. Zhang, T. Bourdel, F. Chevy, and C. Salomon, and we acknowledge the support of the Netherlands Organization for Scientific Research (NWO). E. K. acknowledges support from the Stichting FOM, financially supported by NWO.
[1] C. C. Bradley et al., Phys. Rev. Lett. 75, 1687 (1995); 79, 1170 (1997).

[2] H. Feshbach, Ann. Phys. (N.Y.) 5, 357 (1958); 19, 287 (1962).

[3] L. Khaykovich et al., Science 296, 1290 (2002).

[4] K. E. Strecker et al., Nature (London) 417, 150 (2002).

[5] P. Nozières and S. Schmitt-Rink, J. Low Temp. Phys. 59, 195 (1982); M. Randeria and references in Bose-Einstein Condensation (Cambridge University Press, Cambridge, 1995).

[6] C. A. Regal, M. Greiner, and D. S. Jin, Phys. Rev. Lett. 92, 083201 (2004).

[7] M. W. Zwierlein et al., Phys. Rev. Lett. 92, 120403 (2004).

[8] C. A. Stan, M. W. Zwierlein, C. H. Schunck, S. M. F. Raupach, and W. Ketterle, Phys. Rev. Lett. 93, 143001 (2004).

[9] S. Inouye, J. Goldwin, M. L. Olsen, C. Ticknor, J. L. Bohn, and D. S. Jin, Phys. Rev. Lett. 93, 183201 (2004).

[10] B. M. Smirnov and M. S. Chibisov, Zh. Eksp. Teor. Fiz. 48, 939 (1965) [Sov. Phys. JETP 21, 624 (1965)].

[11] W. T. Zemke and W. C. Stwalley, J. Chem. Phys. 111, 4962 (1999).

[12] M. Marinescu and A. Dalgarno, Z. Phys. D: At., Mol. Clusters 36, 239 (1996).

[13] C. J. Lorenzen and K. Niemax, J. Phys. B 15, L139 (1982).

[14] Z.-C. Yan et al., Phys. Rev. A 54, 2824 (1996).

[15] A. J. Moerdijk and B. J. Verhaar, Phys. Rev. Lett. 73, 518
(1994).

[16] F. A. van Abeelen, B. J. Verhaar, and A. J. Moerdijk, Phys. Rev. A 55, 4377 (1997).

[17] X. Wang et al., J. Chem. Phys. 117, 9339 (2002).

[18] K. M. O'Hara et al., Phys. Rev. A 66, 041401(R) (2002).

[19] S. Jochim et al., Phys. Rev. Lett. 89, 273202 (2002).

[20] K. E. Strecker, G. B. Partridge, and R. G. Hulet, Phys. Rev. Lett. 91, 080406 (2003).

[21] K. M. O’Hara et al., Phys. Rev. Lett. 85, 2092 (2000).

[22] E. R. I. Abraham et al., Phys. Rev. A 55, R3299 (1997).

[23] A. Derevianko, J. F. Babb, and A. Dalgarno, Phys. Rev. A 63, 052704 (2001).

[24] Here $C^{e x}$ is taken from Ref. [11].

[25] J. Zhang et al., Phys. Rev. A 70, 030702 (2004).

[26] E. R. I. Abraham et al., Phys. Rev. Lett. 74, 1315 (1995).

[27] E. v. Kempen et al., Phys. Rev. Lett. 88, 093201 (2002).

[28] B. Marcelis, E. G. M. van Kempen, B. J. Verhaar, and S. J. J. M. F. Kokkelmans, Phys. Rev. A 70, 012701 (2004).

[29] S. De Palo, M. L. Chiofalo, M. J. Holland, and S. J. J. M. F. Kokkelmans, Phys. Lett. A 327, 490 (2004).

[30] D. S. Petrov, Phys. Rev. Lett. 93, 143201 (2004).

[31] L. Viverit, Phys. Rev. A 66, 023605 (2002).

[32] M. Mackie et al., Phys. Rev. A 69, 053614 (2004).

[33] D. S. Petrov, C. Salomon, and G. V. Shlyapnikov, Phys. Rev. Lett. 93, 090404 (2004). 